\title{
The Importance of Human Exploration in Accomplishing High Priority Lunar Science Objectives
}

\section{A LEAG-sponsored white paper for submission to the 2023-2032 Decadal Survey on Planetary Science and Astrobiology}

Lead Author: Kelsey E. Young, NASA Goddard Space Flight Center, kelsey.e.young@ nasa.gov.

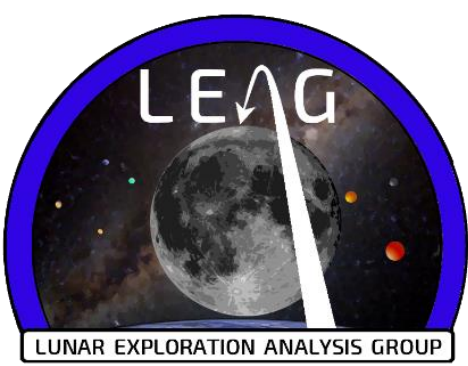

Co-Authors: José M. Hurtado, Jr. (The University of Texas at El Paso), Trevor G. Graff (Jacobs, NASA Johnson Space Center), Daniel P. Moriarty (USRA, NASA Goddard Space Flight Center), Sarah Valencia (University of Maryland, College Park, NASA Goddard Space Flight Center), Ryan Watkins (Planetary Science Institute), Noah Petro (NASA Goddard Space Flight Center), Kirby Runyon (Johns Hopkins University Applied Physics Laboratory), Zachary R. Morse (Howard University, NASA Goddard Space Flight Center), Amy Fagan (Western Carolina University), Clive R. Neal (University of Notre Dame), and Jessica Watkins (NASA Johnson Space Center)

Co-Signers: Barbara A. Cohen (NASA Goddard Space Flight Center), Kurt Retherford (Southwest Research Institute), Joshua Cahill (Johns Hopkins University Applied Physics Laboratory), Briony Horgan (Purdue University), Heather M. Meyer (Johns Hopkins University Applied Physics Laboratory), Brett Denevi (Johns Hopkins University Applied Physics Laboratory), Kerri Donaldson Hanna (University of Central Florida), Donald C. Barker (NASA Johnson Space Center), Ross Potter (Brown University), Maria Banks (NASA Goddard Space Flight Center), Ernie Bell (University of Maryland College Park), Devanshu Jha (MVJ College of Engineering), Renee Weber (NASA Marshall Space Flight Center), Esther Beltran (University of Central Florida/Florida Space Institute), Ashley Royce (University of Central Florida Florida Space Institute), Sean Gulick (University of Texas at Austin), Karl Hibbitts (Johns Hopkins University Applied Physics Laboratory), Cameron M. Mercer (Catholic University, NASA Goddard Space Flight Center), Natalie Curran (Catholic University, NASA Goddard Space Flight Center), Barbara Frasl (Catholic University, NASA Goddard Space Flight Center), Samuel Lawrence (NASA Johnson Space Center), Elizabeth Rampe (NASA Johnson Space Center), Jacob Richardson (University of Maryland College Park, NASA Goddard Space Flight Center), Kaizad Raimalwala (Mission Control Space Services Inc.), Melissa Battler (Mission Control Space Services Inc.) 


\subsection{Introduction}

For many reasons, the Moon is an oasis of scientific discoveries waiting to be explored (Moriarty et al., 2020). The Moon is both the only other planetary body humans have visited and is the target of NASA's upcoming plans for human exploration with the Artemis program. The samples collected and the observations made during the six Apollo lunar surface missions continue to provide ground-breaking science advancements even several decades later (e.g., Saal et al., 2008; Jolliff and Robinson, 2019). Although orbital, lander, and robotic missions are critical in closing scientific knowledge gaps, the scientific advances accomplished through human exploration are extensive and cannot be underestimated. Human cognition, mobility, dexterity, and a host of other factors, are all dynamic variables brought to scientific exploration through the presence of astronauts, which we explore in this white paper. First and foremost, the early integration of the science and human spaceflight communities is critical to mission success. In light of NASA's plans for lunar crewed exploration, the 2023-2032 Planetary Science Decadal Survey should include recommendations for lunar decadal science that would be enhanced by human exploration. The absence of such a recommendation may negatively impact the ability for science to be integrated into Artemis. If a sustained lunar surface presence is going to result from Artemis, science must be a priority, and a Decadal recommendation will support that result.

\subsection{Lunar Science Objectives Overview}

Several community and NASA documents (i.e. Scientific Context for the Exploration of the Moon, 2007; LEAG Lunar Exploration Roadmap, 2016; Global Exploration Roadmap, 2018, 2020; Advancing Science of the Moon, 2018; Artemis Program Lunar Science Objectives, 2020) have established overarching lunar science objectives, which will be solidified by this Planetary Science Decadal Review. Many of these objectives include those that will benefit from the variables explored below. Specifically, the Artemis Program Lunar Science Objectives (2020) include: Studying Planetary Processes; Understanding Volatile Cycles; Determining the Impact History of the Earth-Moon System; Accessing a Record of the Ancient Sun; Conducting Fundamental Lunar Science; and Using the Moon as a Platform to Study the Universe.

\subsection{Variables of Human Exploration}

Discussions about the involvement of humans in planetary exploration are often complex and, in some cases, driven by arguments regarding the relative effectiveness of humans versus robots. Robotic exploration has been crucial for planetary exploration, and the technologies and procedures for carrying it out are highly sophisticated and productive. Robotic exploration can extend the human reach to other worlds, but the human cognitive element is greatly magnified by astronauts being on site. As NASA's plans to return astronauts to the Moon are realized, the science community has the opportunity to take advantage of highly-trained human explorers present, first-hand, on the Moon who can be equipped, both in training and tools, to achieve science objectives to understand Solar System processes. The capacity afforded by humans for doing science can be immense compared to even the most advanced robotic systems. When conducting science remotely, humans are not able to fully exploit their unique capabilities, such as (1) rapid decision making, particularly by trained individuals who can act independently; (2) pattern recognition and intuition; and (3) nimble mobility, all three of which contribute to serendipitous discovery (e.g., Apollo 15 "seatbelt rock" and the Apollo 17 orange soil). 


\subsection{Discovery-Based Science and Operational Flexibility}

The value of putting a trained human scientist with the ability to react real-time to in situ observations on the lunar surface is an extensive benefit to accomplishing lunar decadal science. An astronaut's binocular vision and natural predisposition for pattern recognition and the ability to make observations and contextualize them rapidly in 3D space, paired with rapid decision making and their more versatile movement capabilities, makes them better at identifying geologically significant targets that might otherwise be missed by a robotic explorer.

The value of rapid decision-making manifests in many ways. For example, humans have the ability to rapidly sense and then react to changing operational and environmental conditions, allowing a human explorer to identify, avoid, and mitigate potential hazards in real time.

Similarly, a human explorer can recognize, decide, and act upon new scientific observations very rapidly. Communication lags, among other technological limitations, restrict the speed at which a robotic asset can react to similar factors. The trade, then, is that the pace of robotic exploration is often necessarily far slower than what a human could achieve. The result is that, as measured over an equal amount of time, a human explorer has the capacity to see more, go farther, and do more than a robot, potentially yielding more science return.

The ability to recognize patterns, or deviations from them, to achieve continuous situational awareness of the environment and of science progress is the foundation of doing field science. While advances in artificial intelligence are making strides in enabling machines in this regard (e.g., machine vision in autonomous vehicles), the human element is still important for using the output for higher-level decision making. It is one thing to design a rover to autonomously sense and avoid a hazard of known form, such as a boulder. It is quite another for a rover, by itself, to realize that the boulder in its way may be a piece of South Pole-Aitken Basin (SPA) ejecta and that it looks to contain a xenolith of lunar mantle material. Having human cognition, curiosity, and intuition intrinsically linked to the sensing and pattern recognition is key, and the human brain and visual system remain the state-of-the-art. This capability can be fully exploited and transformative when the human is present in the field, rather than on-console, far away.

\subsection{Dexterity}

Robotic missions, such as those exploring the surface of Mars, have made important scientific advancements, yet these rovers are limited in their ability to traverse steeply sloping or rockstrewn terrain, access outcrops, and collect samples of interest. While several current and upcoming robotic missions do have robotic arms capable of collecting samples and providing close approaches to outcrops of interest, astronauts are able to autonomously navigate hummocky and rocky terrains as well as approach and sample boulders and outcrops that often prevent obstacles to wheeled vehicles. For example, Apollo 17 astronauts were able to circumnavigate a series of five large boulders at station 6 , taking samples from various lithologies from multiple spots around the boulders and also from soils and shadowed regions near and under the boulders. This activity yielded valuable science data and would prove either impossible or incredibly time-consuming to do with a robotic explorer (Meyer, 2010). Sample return is vital to answering high priority lunar science objectives (Valencia et al., 2020), and the dexterity afforded by human exploration will maximize the effectiveness of that sample return.

Additionally, the ability of a robotic rover or lander to manipulate smaller, more complex samples and payloads is not comparable to a suited astronaut's dexterous capability. Several of the community-driven high priority lunar science objectives either specifically call out or would benefit from the deployment and manipulation of delicate science payloads or complex sampling 
tools. These payloads can be deployed and calibrated by astronauts (e.g., delicate astrophysics telescope on the radio quiet lunar far side) or used in situ by astronauts (e.g., handheld analytical tools) to get real-time data to inform sample collection and traverse execution.

\subsection{The Importance of Mobility}

Particularly for field geology, where often the most interesting and important study sites are also the most difficult to reach, mobility assets that allow astronauts to travel greater distances and access a diversity of terrains are a major enabling factor. The nature of the scientific questions, and the materials required to answer those questions, often results in the key outcrops, from which observations and samples are desired, to be on steep slopes or cliffs, within subsurface cavities (e.g. caves, lava tubes), or across rough and uneven terrain.

Astronauts on the Moon, even without mobility assets such as an unpressurized or pressurized rover, are valuable as they are able to precisely maneuver to targets of interest and move much faster than a robotic explorer (Figure 1). As shown by the Apollo J missions (Apollo 15-17; Figures 2 and 3), adding mobility assets such as the Lunar Roving Vehicle (LRV) to a human exploration mission further increases the science return by allowing the crew to cover more ground and access more challenging locations, resulting in an increase in visual observations, photo-documentation, and sample diversity. In addition, having mobility assets, like the LRV, increases the capacity for carrying equipment, particularly over long distances. This capability also increases the number, mass, and/or volume of samples that can be gathered from a field area. While the Apollo J missions landed in relatively smooth environments, the addition of the LRV enabled access to what would have been difficult or impossible locations on which to land.

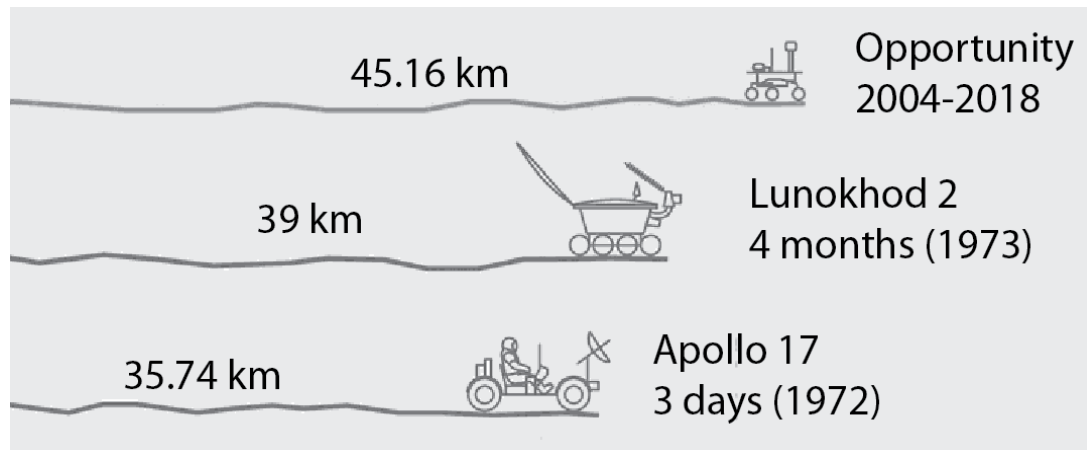

Figure 1. Comparison of

traverse distances for

Opportunity on Mars, Lunokhod

2 on the Moon, and Apollo 17

(equipped with the LRV). They

travelled similar distances, but

the robotic rovers did so in much

longer time. Modified from

original NASA/JPL-Caltech

infographic.

Many of the high priority lunar science questions highlighted above and discussed in detail in numerous community documents and white papers benefit strongly from mobility in that a rover to assist astronauts would allow access to a broader diversity of terrain. Specifically, returning samples from the lunar surface is essential for addressing several of the highest-priority science and exploration goals, as sample return enables detailed chemical and physical analyses possible only in terrestrial laboratories. At present, humans (Apollo 11, 12, 14-17) have returned $382 \mathrm{~kg}$ of lunar samples, while robotic missions (Soviet Luna 16, 20, and 24) returned a total of 301 grams. While both approaches have their value, the amount and diversity of the Apollo collection is unparalleled relative to robotic sample return missions.

Typically, different science goals require sampling specific lithologies corresponding to specific areas on the lunar surface. Different lithologies exhibit different surface distributions, meaning that each science goal dictates the need to collect samples from different areas of the Moon. Furthermore, several science questions related to unraveling geologic history and compositional diversity benefit from analysis of multiple sample types from multiple locations. Also, samples 
are the "gift that keeps on giving" and can be used to address new science questions long after they have been returned (e.g., Pernet-Fisher et al., 2019). Therefore, addressing the full suite of high-priority science goals established by the planetary science community necessitates sampling a diversity of materials from multiple locations on the lunar surface.

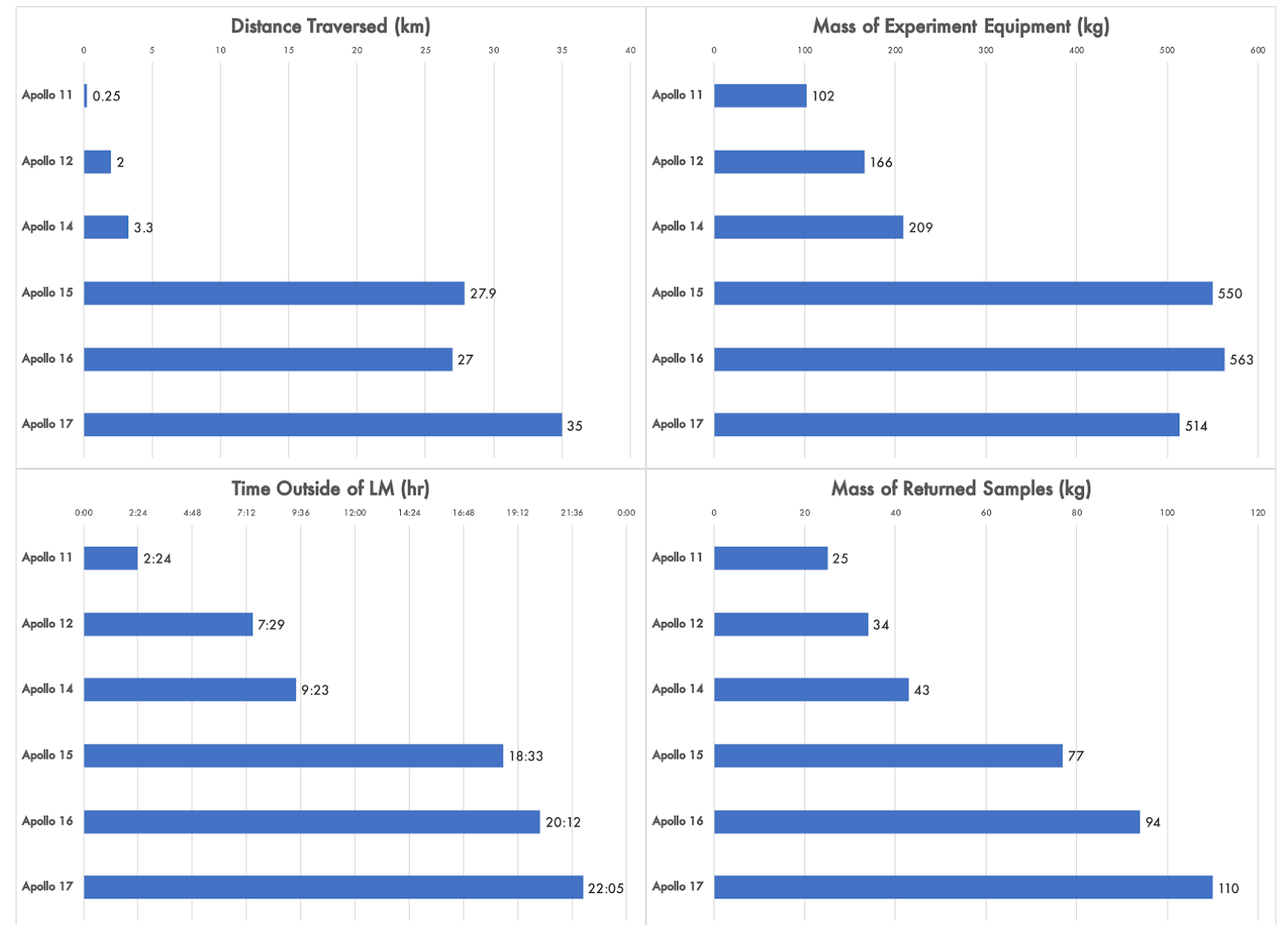

Figure 2: Data from the Apollo 17 Preliminary Science Report (1973) illustrates the value of increased human exploration capability on science return. Science return is quantified with the metrics: distance traversed (longer distance $=$ more ability to explore new terrains and increase sample diversity); mass of experiment equipment (increased mass = more capability to accomplish science objectives); time outside the lunar module $(L M)$ (more time = more opportunities for science discovery); and mass of returned samples (increased mass = more samples for scientists to study back on Earth). All three metrics dramatically increased with the J missions (Apollo 15-17).

As the return of US assets to the Moon moves closer to reality, the scientific community has identified desirable landing sites that would be useful in retiring outstanding science questions and strategic knowledge gaps (Jawin et al., 2019). As a part of this process, the lunar community met for the Lunar Science for Landed Missions workshop in January, 2018. The workshop result was a set of high-priority landing sites that will advance the fields of lunar and Solar System science. The workshop also considered the role of mobility in achieving priority science and exploration goals. The message was clear: mobility was either enabling or enhancing for the vast majority of landed lunar missions desired by the lunar science community (Jawin et al., 2019). 

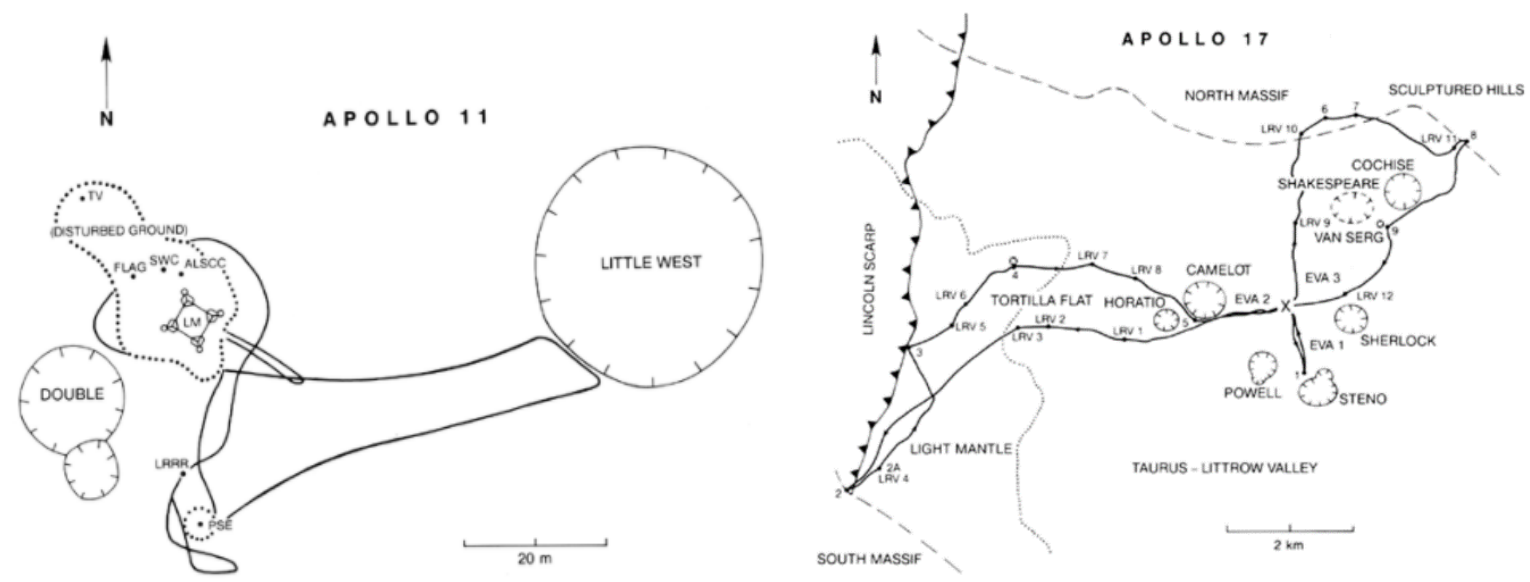

Figure 3. Comparison of traverses for Apollo 11 (left) with traverses for Apollo 17 (right). Apollo 17, one of the J missions, was equipped with the Lunar Roving Vehicle (LRV). Note the factor of 100 difference in scale between the two maps. Maps from the Lunar and Planetary Institute (www.lpi.usra.edu/publications/slidesets/apollolanding/).

\subsection{Use Case: Sampling the South Pole-Aitken (SPA) Basin}

Several of the highlighted high priority lunar surface objectives are dependent on navigation to disparate sites of interest identified from remotely sensed data. Some of these objectives are also dependent on the ability to extract specific sections of these identified outcrops. For example, Lunar Reconnaissance Orbiter (LRO) and Moon Mineralogy Mapper ( $\mathrm{M}^{3}$ ) data have shown that SPA has tapped a variety of pre-impact stratigraphic units that may be preserved in uplifted material within the basin (Moriarty and Pieters, 2018, Ohtake et al., 2014). Navigating to these specific areas to extract samples of these unique mafic materials will require astronauts to map a region, contextualize their observations in relation to this very complex scientific question, possibly use in situ analytical instrumentation to identify high priority material, and extract the requisite samples. The age of the SPA is one of the highest priority lunar and Solar System science questions. Targeting this question with astronauts yields the highest likelihood of constraining the timing of the SPA impact, a critical cornerstone for understanding absolute lunar chronology and the bombardment history of the inner solar system.

\subsection{Integrating the Scientific and Human Spaceflight Communities}

\subsection{The Legacy of Apollo}

The planning and lessons learned from Apollo in conducting science operations must be leveraged as we move forward with crewed planetary surface exploration. One of these lessons, recognized early in planning for Apollo, is the importance of the relationship between the scientific and human spaceflight communities. Between geologists providing field training to the Apollo astronauts, scientists populating the science backroom during Apollo operations, and scientist-astronauts making an impact on the evolution of the Apollo program (culminating in geologist Harrison Schmitt conducting science on the lunar surface during Apollo 17), the Apollo program demonstrated the value of creating a shared culture and language of scientific exploration among the entire exploration community. As we move forward toward the next generation of crewed space exploration, this same collaboration and integration is critical to the 
success of Artemis. Ensuring that scientists are engaged and included in mission planning and engineering decisions is crucial and needs to start as soon as possible.

\subsection{The Importance of Integrated Testing in Analog Environments}

Testing tools and instruments for sample and data collection, evaluating procedures and conops for lunar surface science, and training astronauts, engineers, flight controllers, and scientists in conducting surface operations are all tasks that can and should be accomplished at planetary analog sites, or locations that resemble another planetary surface. In addition to being used to understand planetary processes, testing at analog sites is a necessary step as we prepare for crewed lunar exploration, and eventual martian exploration (Whelley et al., 2020).

\subsection{Conclusions}

The intersection of the human capabilities discussed above (human cognition, dexterity, and mobility) enables -- particularly for trained and experienced field scientists -- practice of the scientific method in the integrated way described by Hodges and Schmitt (2011) wherein the processes of planning, execution, and re-planning of tasks are intrinsically linked to the processes of observation, description/documentation, and decision-making to develop and test multiple working hypotheses. This "flexecution" process can be (and is) done during robotic exploration, but having in situ human presence greatly accelerates the pace and increases the efficiency of planetary surface mission operations. While it is challenging to measure the increase in science return from an astronaut completing science objectives in situ, metrics such as distance traveled, returned sample mass, exploration and traverse time, and science payloads deployed (Figure 2), all help track the potential for future human explorers to make significant advances in the Planetary Science Decadal Survey objectives which will help us unravel critical lessons learned about our nearest celestial neighbor.

In addition to the scientific benefits explored in this white paper, human explorers are also uniquely qualified to engage members of the public and the future of the lunar science community by conveying their experiences, feelings, and discoveries made during lunar exploration. This human capacity to lift up and inspire both the public and the next generation of scientists and explorers is yet another benefit to human exploration.

\subsection{Recommendations for the 2023-2032 Planetary Science Decadal Survey}

\subsubsection{Recommendation \#1: The lunar science community should be integrated with the human spaceflight community to ensure adequate understanding of lunar science} objectives and criteria for success. A critical lesson learned from the Apollo program was the importance of integrating the scientific and human spaceflight communities. As scientists, we must understand the constraints and risks involved with flying humans in space and returning them safely to Earth. We must also make it a priority to teach our human spaceflight colleagues about the fundamentals of the Moon and of lunar science objectives. This is important to ensure that they have an accurate understanding of what to expect of the lunar surface and so they can take partial ownership over our science objectives to ensure those objectives are prioritized in crewed mission planning and execution. This should include working meetings and workshops designed to facilitate the exchange of ideas as well as continued analog testing involving both communities. Funding should be provided for scientists to participate in these analog activities and to integrate with the human spaceflight community, as this is critical work that is not adequately funded in the current fiscal environment. 


\subsubsection{Recommendation \#2: The Planetary Science Decadal Survey should include a}

strategy for maximizing lunar science through human exploration, not just through the more traditional orbital and landed missions. Although prior Decadal Surveys have addressed mission/measurement recommendations for accomplishing high priority lunar science, these surveys have not traditionally addressed recommendations for how best to accomplish science using human exploration. Given NASA's current Artemis plans for lunar exploration, we recommend the 2023-2032 Decadal Survey include links where possible between the Decadal Survey science to the possibilities afforded to the science community with human exploration.

\subsection{References}

Advancing Science of the Moon: Report of the Specific Action Team (February 2018). Lunar Exploration Analysis Group (LEAG), www.lpi.usra.edu/leag.

Apollo 17 Preliminary Science Report (1973). National Aeronautics and Space Administration (NASA), NASA SP-330. www.hq.nasa.gov/alsj/a17/as17psr.pdf.

Artemis Program Lunar Science Objectives (May 2020). NASA, presented at the Lunar Surface Science Workshop (LSSW), www.hou.usra.edu/meetings/lunarsurface2020.

Global Exploration Roadmap 3rd edition (January 2018). International Space Exploration Coordination Group (ISECG), www.globalspaceexploration.org.

Global Exploration Map Supplement: Lunar Surface Exploration Scenario Update (August 2020). ISECG, www.globalspaceexploration.org.

Hodges, K.V. and H. H. Schmitt (2011). A new paradigm for advanced planetary field geology developed through analog experiments on Earth, in Garry and Bleacher, eds. Analogs for Planetary Exploration: GSA Spec. Paper 483, p. 17-31, doi:10.1130/2011.2483(02).

Jawin, E. R., et al. (2019). Lunar Science for Landed Missions Workshop Findings Report. Earth and Space Science, 6(1), 2-40.

Jolliff, B. L., \& Robinson, M. S. (2019). The Scientific Legacy of the Apollo Program. Physics Today, 72(7), 44-50. https://doi.org/10.1063/PT.3.4249.

Meyer, C. The Lunar Sample Compendium. 2010. See 76031,76240.

Moriarty, D. P., \& Pieters, C. M. (2018). The character of south pole-Aitken basin: patterns of surface and subsurface composition. JGR: Planets, 123(3), 729-747.

Moriarty, D.P., C. Neal, S. Lawrence, et al. (2020). The Moon is a Special Place. White Paper for the 2023-2032 Decadal Survey on Planetary Science and Astrobiology.

Ohtake, Makiko, et al. "Geologic structure generated by large-impact basin formation observed at the South Pole-Aitken basin on the Moon." GRL Letters 41.8 (2014): 2738-2745.

Pernet-Fisher J. F., et al. (2019). 50 years on: legacies of the Apollo programme. Astronomy \& Geophysics, 60(4), 22-28.

Saal, Alberto E., Erik H. Hauri, Mauro L. Cascio, James A. Van Orman, Malcolm C. Rutherford, and Reid F. Cooper. "Volatile content of lunar volcanic glasses and the presence of water in the Moon's interior." Nature 454, no. 7201 (2008): 192-195.

The Scientific Context for Exploration of the Moon (2007). National Research Council. The National Academies Press. https://doi.org/10.17226/11954.

The Lunar Exploration Roadmap: Exploring the Moon in the 21st Century: Themes, Goals, Objectives, Investigations, and Priorities (2016), LEAG, v1.3, www.lpi.usra.edu/leag.

Valencia, S.N. et al. (2020). High Priority Returned Lunar Samples. White Paper for the 20232032 Decadal Survey on Planetary Science and Astrobiology.

Whelley, P.L. et al. (2020), The Importance of Field Studies to Closing Key Knowledge Gaps in Planetary Science. White Paper for the 2023-2032 Decadal Survey on Plan. Sci. \& Astro. 